\title{
Aproximación conceptual al sexismo ambivalente: Estado de la cuestión
}

\section{Conceptual approach to ambivalent sexism}

\author{
Yolanda Rodríguez Castro¹, María Lameiras Fernández, \\ María Victoria Carrera Fernández, Jose María Faílde Garrido \\ Universidad de Vigo, España
}

(Recepción: Octubre 2009 - Aceptación: Diciembre 2009)

\begin{abstract}
Resumen
El objetivo de este artículo es abordar los fundamentos de la teoría del sexismo ambivalente, tanto de las escalas desarrolladas para mujeres (ASI, Escala de Sexismo Ambivalente, Glick y Fiske, 1996) como para hombres (AMI, Escala de Actitudes Ambivalentes hacia hombres, Glick y Fiske, 1999) y su relación con otras escalas de sexismo moderno; así como las repercusiones de la manifestación de este nuevo sexismo en las investigaciones nacionales e internacionales. Los resultados confirman que la escala ASI es la única que mide un sexismo relacional frente a las escalas de Neosexismo (Tougas et al., 1995) y de MOS (Swim et al., 1995) que miden un sexismo socio-laboral. También se evidencia la repercusión que la escala ASI ha tenido en la comunidad científica en detrimento de la escala AMI.
\end{abstract}

Palabras clave: sexismo ambivalente, hostilidad, benevolencia, género.

\begin{abstract}
The aim of this article is to study ambivalent sexism theory, and the scales of Ambivalent Sexism Inventory (ASI; Glick y Fiske, 1996) and Ambivalent Attitudes toward Men (AMI, Glick y Fiske, 1999). We study the relationship with other sexism' scales. The results show that ASI measure sexism relatonship and Neosexism Scale (Tougas et al., 1995) and the MOS Scale (Swim et al., 1995) measure a work-social. The ASI have more important in cientific comunity than the AMI.
\end{abstract}

Key-words: Ambivalent sexism, hostility, benevolence, gender.

1 Correspondencia: Yolanda Rodríguez Castro. Facultad de Ciencias de la Educación, Av. Castelao sn, 32004. Pabellón 2. Campus de Ourense, Universidad de Vigo, España. E-mail: yrcastro@uvigo.es 
Las conceptualizaciones más tradicionales del sexismo basadas en las diferencias biológicas entre hombres y mujeres, han ido involucionando al mismo tiempo que emerge otra cara más moderna del sexismo, que se nutre de los nuevos postulados surgidos en torno al racismo moderno (Sears, 1988). Postulados que se inician de la mano de Tougas, Brown, Beaton, y Joly (1995) y Swim, Aikin, HaII y Hunter (1995) parten de la idea que junto a los sentimientos negativos hacia las mujeres, que perviven de las formas más tradicionales de sexismo, el sexismo moderno convive la aceptación de valores igualitarios, socialmente deseables en aquellas sociedades que han evolucionado hacia posicionamientos más liberales. Planteando el estudio del sexismo desde la dimensión social y estableciendo como señas de identidad las barreras al avance de la mujer en el mundo público, y la considerada excesiva condescendencia que se supone a las acciones positivas.

Glick y Fiske $(1996,1999)$ defienden, desde una propuesta más reciente y en un intento de avanzar en una mejor comprensión del sexismo moderno, que para el estudio de este es necesario incorporar parámetros explicativos que surgen de la dimensión relacional. Lo que implica que las relaciones entre los sexos no pueden ser articuladas exclusivamente desde una perspectiva intergrupal, y supone reconocer que frente a la visión de los sexos como grupos en un contexto social sometidos a fuerzas divergentes de independencia y autonomía, estos están necesariamente vinculados en un mundo relacional de fuerzas convergentes de dependencia y heteronomía. La combinación de estas fuerzas centrífugas y centrípetas son las que articulan la constelación de actitudes hacia los sexos.

Este nuevo sexismo lo denominan Glick y Fiske (1996) Sexismo Ambivalente, que se define como el resultado de la combinación de dos elementos con cargas afectivas antagónicas, que son consecuencia de las complejas relaciones de aproximación/evitación que caracteriza a los sexos. En este sentido, los autores desarrollaron inicialmente un instrumento para evaluar el sexismo de los hombres hacia las mujeres que denominaron Escala de Sexismo Ambivalente (ASI: Glick y Fiske, 1996). Posteriormente, Glick y Fiske (1999) elaboraron la Escala de Ambivalencia hacia hombres (AMI). Esta escala es la hermana de la escala ASI, y ambas reflejan las actitudes ambivalentes hacia los sexos que son resultado del poder estructural de los hombres y del poder diádico de las mujeres.

Por lo tanto, a lo largo de este artículo abordaremos los fundamentos de la teoría ambivalente, junto con el estudio de las escalas de sexismo ambivalente hacia mujeres y hombres y su relación con otras escalas de sexismo; así como las repercusiones de la manifestación de este nuevo sexismo en las investigaciones nacionales e internacionales.

\section{Actitudes ambivalentes hacia mujeres}

En 1996 Glick y Fiske introducen el concepto de Sexismo Ambivalente resultado de la coexistencia de afectos y actitudes positivas y negativas hacia las mujeres. Este tipo de sexismo hace referencia a la combinación de dos elementos con cargas afectivas antagónicas, que son consecuencia de las complejas relaciones que caracteriza a los sexos.

Glick y Fiske (1996) argumentan que la tradición literaria sobre sexismo lo ha conceptualizado como un reflejo de la hostilidad hacia las mujeres pero omite un aspecto significativo del sexismo que son los sentimientos subjetivamente positivos hacia las mujeres que suelen ir relacionados con una visión negativa hacia las mujeres. Glick y Fiske (1996) contemplan el sexismo como un constructo multidimensional que abarca dos tipos de actitudes sexistas: Sexismo Hostil y Sexismo Benevolente.

El Sexismo Hostil coincide básicamente con el viejo sexismo, al que aludía Allport (1954) en su clásica definición de prejuicio, entendido como una antipatía. Mientras que el Sexismo Benevolente se define como un conjunto de actitudes interrelacionadas hacia las mujeres que son sexistas en cuanto las considera de forma estereotipada y limitadas a ciertos roles, pero que tiene un tono afectivo positivo para el preceptor y tiende a suscitar en éste conductas típicamente categorizadas como 
prosociales (de ayuda) o de búsqueda de intimidad (como la revelación de uno mismo) (Glick y Fiske, 1996). Este Sexismo Benevolente no es bueno debido a que enmascara actitudes prejuiciosas hacia las mujeres, y puede despistar a las mujeres con su tono afectivo y positivo.

Para entender esta teoría del sexismo ambivalente es necesario partir de la posición teórica de la ambivalencia propuesta por Katz (1981) y por Katz y Hass (1988) Estos autores analizan un supuesto conflicto entre las dos orientaciones axiológicas de los norteamericanos que son el igualitarismo y el individualismo. Estas dos orientaciones pueden producir un conflicto y generar ambivalencia de sentimientos y actitudes dentro de las personas.

Siguiendo esta misma línea argumental, Glick y Fiske (1996) parten de que la ambivalencia sexista se origina en la influencia simultánea de dos tipos de creencias sexistas porque son dos constructos subjetivamente vinculados a sentimientos opuestos hacia las mujeres. El hombre sexista puede ser ambivalente en el sentido de que sus actitudes indican una tendencia a responder de forma muy favorable o totalmente desfavorable hacia las mujeres. Aunque los hombres no experimentan conflictos según Glick, Diebold, Bailey-Werner, y Zhu (1997) porque el sexismo ambivalente es capaz de reconciliar las creencias sexistas hostiles y las benevolentes sin sentimientos conflictivos y esto lo sugiere la alta correlación entre el Sexismo Hostil y Sexismo Benevolente (Glick y Fiske, 1996).

Los hombres sexistas evitan conflictos entre sus actitudes positvas y negativas hacia las mujeres, clasificando a las mujeres en subgrupos, uno bueno y otro malo, en los que se incluyen aspectos positivos y negativos del sexismo ambivalente. Glick et al. (1997) sugieren que si cada mujer puede categorizarse rápidamente dentro de un subgrupo, favorable o desfavorable, los hombres no tendrían sentimientos confusos o conflictivos hacia ellas y que el sexismo ambivalente estaría constituido por ideas claras y consistentes. Porque para los hombres sexistas existen grupos de mujeres que merecen un tratamiento hostil, mientras que otros grupos de mujeres merecen ser tratadas con benevolencia.

Por tanto, el sexismo ambivalente crea visiones polarizadas de subtipos de mujeres. El Sexismo Hostil se aplica como un castigo a las mujeres no tradicionales como mujeres profesionales y feministas porque estas mujeres no asumen los roles de género tradicionales e intentan alterar las relaciones de poder entre hombres y mujeres. Mientras que el Sexismo Benevolente es una recompensa a las mujeres que cumplen los roles tradicionales porque estas mujeres aceptan la supremacía masculina. Por consiguiente, el Sexismo Hostil y el Sexismo Benevolente actúan como un sistema articulado de recompensas y castigos con la finalidad de que las mujeres sepan cual es su posición en la sociedad.

De esta manera, Glick y Fiske (1996, 1999) sugieren que, tanto el Sexismo Hostil como el Benevolente, giran alrededor del poder social, la identidad de género y la sexualidad. Así, los autores proponen que el Sexismo Hostil y el benevolente se articulan entorno a tres componentes comunes: el Paternalismo, la Diferenciación de Género y la Heterosexualidad. Cada componente refleja una serie de creencias en las que la ambivalencia a las mujeres es inherente, ya que cada componente tiene un aspecto hostil y otro benévolo; y sirve para justificar las condiciones sociales y biológicas que caracterizan las relaciones entre sexos.

1.- El Paternalismo: Glick y Fiske (1996) lo definen como la forma en que un padre se comporta con sus hijos/as, por un lado les aporta afecto y protección y por el otro el padre es el que manda sobre sus hijos/as. Esta concepción está íntimamente relacionada con la visión ambivalente del sexismo, porque incluye dos dimensiones: el paternalismo protector y el paternalismo dominador. El sexismo se materializa en paternalismo dominador que se asienta en la estructura del patriarcado que legitima la superioridad de la figura masculina. Por otro lado, el sexismo también se materializa en un paternalismo protector, que los hombres aplican a las mujeres que desempeñan roles tradicionales, ya que las consideran como criaturas débiles y frágiles a las que hay que colocar en un pedestal y proteger (Glick y Fiske, 1996).

2.- La Diferenciación de Género: para Glick y Fiske (1996) la diferenciación de género constituye el segundo componente en el que subyace el Sexismo Hostil y el Sexismo Benevolente. En la 
diferenciación de género también nos encontramos con las dos caras del sexismo: por un lado está la diferenciación de género competitiva que se presenta como una justificación sobre el poder estructural masculino y por el otro la diferenciación de género complementaria (Glick y Fiske, 1996). Por otro lado, los hombres son conscientes del poder diádico de las mujeres que les hace depender de ellas. Este poder hace que los hombres reconozcan que las mujeres tienen características positivas (Eagly y Mladinic, 1993) que complementan a las suyas. Esto es lo que constituye la diferenciación de género complementaria.

3.- La Heterosexualidad: para Glick y Fiske (1996) constituye uno de los más poderosos orígenes de la ambivalencia de las actitudes de los hombres hacia las mujeres. Al igual que los anteriores componentes, la heterosexualidad tiene dos vertientes: la intimidad heterosexual y la hostilidad heterosexual. Glick y Fiske (1996) establecen que la motivación sexual de los hombres hacia las mujeres puede estar unida a un deseo de proximidad (intimidad heterosexual). La dependencia diádica de los hombres respecto a las mujeres crea una situación bastante inusual en la que los miembros del grupo dominante son dependientes de los miembros del grupo subordinado (Glick y Fiske, 1996). Las mujeres por medio de la sexualidad tienen el poder para satisfacer el deseo de los hombres, en su intimidad heterosexual.

Por consiguiente, Glick y Fiske (1996) afirman que la combinación del Sexismo Hostil con el Sexismo Benevolente constituyen ideologías sexistas complementarias, que son comunes a todas las culturas (Glick et al., 2000) y sirven para mantener las desigualdades entre hombres y mujeres. Es la fuerte correlación entre el Sexismo Hostil y el Sexismo Benevolente (Glick y Fiske, 1996; Glick et al., 2000; Expósito, Moya y Glick., 1998; Lameiras, Rodríguez y Sotelo, 2001; Lameiras y Rodríguez, 2002, Lameiras, Carrera y Rodríguez, 2004; Rodríguez, 2006) la que refuerza la idea de que ambos sexismos son complementarios. Ambas escalas suelen correlacionar habitualmente en un rango mayor a .40, siendo las mujeres las que obtienen correlaciones mayores. Esto puede ser debido a que las actitudes hostiles y las benevolentes de los hombres se diferencian más que las de las mujeres (Glick y Fiske, 1996; Masser y Abrams, 1999; Glick et al., 2000; y Glick et al., 2004; Rodríguez, 2006) porque ellos obtienen puntuaciones mayores en sexismo; es decir, las personas más sexistas suelen conseguir correlaciones más débiles entre el Sexismo Hostil y el Sexismo Benevolente. Otra implicación de la débil correlación entre el Sexismo Hostil y el Sexismo Benevolente entre los participantes más sexistas es que muchos de ellos son más puramente hostiles o benévolos en lugar de ambivalentes (Glick et al., 2000).

Glick et al. (2000) en un estudio transcultural que abarca una muestra de 15.000 hombres y mujeres de 19 naciones de los cinco continentes, reafirman las predicciones hechas en estudios anteriores (Glick y Fiske, 1996; 1997) de que los hombres son más sexistas hostiles que las mujeres. También se confirma que a medida que aumenta el Sexismo Hostil también se incrementa el Sexismo Benevolente, y viceversa. En las sociedades tradicionales el nivel de sexismo es más alto (Glick et al., 2000; Lameiras et al., 2002; Moya, Páez, Glick, Fernández y Poeschl, 2001).

\section{La escala ASI}

La Escala de Sexismo Ambivalente (Glick y Fiske, 1996) fue creada para medir las actitudes ambivalentes de los hombres hacia las mujeres. Esta escala consta de 22 ítems que se agrupan en un único factor de Sexismo Hostil (ítems: 2, 4, 5, 7, 10, 11, 14, 15, 16, 18 y 21) y en tres subfactores de Sexismo Benevolente denominados: Paternalismo protector (ítems: 3, 9, 17, 20); Diferenciación de Género Complementaria (ítems: 8, 19, 22); e Intimidad Heterosexual (ítems: 1, 6, 12, 13). Las fiabilidades obtenidas en las subescalas de Sexismo Hostil y de Sexismo Benevolente son altas, están entre .75 y .90 (Glick y Fiske, 1996, Glick et al., 2000). Actualmente también existe una versión reducida de 12 ítems. Posteriormente Glick y su equipo de investigación validaron la escala ASI en un estudio transcultural en el que contaban con 15.000 participantes de 19 naciones (Glick et al., 2000). Se confirma el modelo factorial completo en 16 de los 19 países, en: España. Holanda, Corea del Sur, Chile, E.E.U.U., Nigeria, Alemania, Brasil, Inglaterra, Turkía, Bélgica, Sudáfrica, Australia, Botswana, Italia y Portugal. Mientras que el modelo de Sexismo Hostil con tres subfactores indepen- 
dientes, igual que en el Sexismo Benevolente, se confirma en Colombia. El modelo de dos factores, se confirma en Japón y Cuba.

Estos resultados apoyaron la hipótesis de que el Sexismo Hostil y el benevolente no son específicos de los EE.UU. sino que son ideologías coherentes que se evidencian a través de las culturas y que el ASI es una medida válida para evaluar estas dimensiones en los distintos países.

\section{Escala ASI y otras medidas de sexismo}

Diversos estudios sobre el Sexismo Ambivalente han confirmado que entre la escala de Sexismo Hostil y la Escala de Sexismo Benevolente, existe una fuerte relación, que sugiere que evalúan ideologías que son complementarias (Glick y Fiske, 1996, 1997; Expósito, Moya y Glick, 1998; Moya et al., 2001; Lameiras et al., 2001; Glick et al., 2000; Lameiras y Rodríguez, 2002; Glick et al., 2004; Rodríguez, 2006). El Sexismo Hostil refleja una visión negativa de las mujeres como deseosas de obtener el poder sobre los hombres, y el Sexismo Benevolente refleja una visión positiva de las mujeres, que las concibe como criaturas débiles a las que hay que proteger.

Todo esto les lleva a Glick y Fiske (1996) a afirmar que la escala ASI es la única escala que conjuga el sexismo tradicional, que considera a las mujeres subordinadas con un nuevo Sexismo Benévolo, que está constituido por un componente afectivo y conductual positivo pero que sigue considerando a la mujer de forma estereotipada y limitada a desempeñar determinados roles tradicionales. Estos autores apuntan que las demás medidas de sexismo moderno como la escala de Neosexismo (Tougas et al., 1995) o la escala de Sexismo Moderno (Swim et al., 1995), no miden las actitudes subjetivamente positivas hacia las mujeres que las sigue limitando a roles tradicionales. Ya que, aunque estas medidas de sexismo moderno pueden correlacionar con la puntuación total del ASI, o incluso con la subescala de Sexismo Benevolente pero que sería explicada por la fuerte correlación que existe entre Sexismo Hostil y Sexismo Benevolente. No obstante, cuando se realizan las correlaciones parciales entre estas escalas y las de Sexismo Benevolente, controlando los efectos de la escala de Sexismo Hostil, no deberían ser significativas.

Glick y Fiske (1996) afirman que la escala de Sexismo Moderno y la de Neosexismo tenían una mayor utilidad predictiva para explorar las actitudes políticas (Medidas de Acción Positiva) relacionadas con el género.

McHugh y Frieze (1997) en su estudio examinan las propiedades psicométricas de seis escalas de sexismo tradicional y de sexismo moderno, entre estas últimas se encuentran la escala de Sexismo Moderno (Swim et al., 1995) y la escala de Sexismo Ambivalente (Glick y Fiske, 1996) junto a la fundamentación teórica. Afirman que las escalas de AWS (Spence y Helmreich, 1972) junto con la de Sexismo Moderno (Swim et al., 1995) se centran en los roles tradicionales de las mujeres en la esfera pública incluyendo trabajo, educación y política; mientras que la escala ASI (Glick y Fiske, 1996) incluye aspectos referidos a la dominancia interpersonal, así como a la heterosexualidad y a las relaciones de intimidad.

Masser y Abrams (1999) a través de tres estudios, confirman que la escala ASI (Glick y Fiske, 1996) y la de Neosexismo (Tougas et al., 1995) son medidas de sexismo moderno, pero que sólo la escala de Sexismo Benevolente permite evaluar el lado subjetivamente positivo del sexismo contemporáneo. De hecho, la subescala de Sexismo Hostil y la escala de Neosexismo miden constructos similares, ya que ambas están significativamente asociadas con actitudes hacia los derechos de las mujeres.

Otro estudio que confirma la hipótesis de Glick y Fiske (1996) es el de Lameiras, Rodríguez, Calado y Carrera (2006) con docentes universitarios gallegos en los que se estudia la relación entre la escala de Neosexismo (Tougas et al., 1995) con la Escala de Sexismo Ambivalente (Glick y Fiske, 1996). A este respecto, los resultados encontrados demuestran nuevamente que el Neosexismo sólo correlaciona con el Sexismo Hostil y no con el Sexismo Benevolente, y solo se produce una pequeña correlación en el grupo de las profesoras. Esto nos puede indicar que la escala de Sexismo Hostil y la de Neosexismo comparten la carga negativa del prejuicio, aunque el Neosexismo se centra en la 
dimensión social-laboral; en ningún caso esta escala detectaría el sexismo encubierto con un tono afectivo positivo como es el Sexismo Benevolente.

Por su parte, Buhl, Hanges, Sipe y Salvaggio (1999) refutan la hipótesis de Glick y Fiske (1996) de que las escalas de sexismo moderno sólo miden el Sexismo Hostil, ya que en su estudio encontraron que la escala de Swim et al. (1995) correlacionaba tanto con la escala de Sexismo Hostil como con la de Sexismo Benevolente.

En esta misma línea se sitúan los resultados de Expósito et al. (1998) con población adulta, cuando compara la escala de Ideología de Género (Moya, Navas, y Gómez Berrocal, 1991) que es una medida de sexismo tradicional, con la de ASI. Los resultados de esta investigación no respaldan totalmente la hipótesis de Glick y Fiske (1996), ya que encontraron que el Sexismo Benevolente continuaba relacionado con la otra medida de sexismo tradicional, incluso controlando la influencia del Sexismo Hostil, aunque la correlación era menor.

Otros estudios con estudiantes de Educación Secundaria Obligatoria (Lameiras y Rodríguez, 2002) y con estudiantes de Educación Secundaria y Universitarios (Lameiras y Rodríguez, 2003; Frese, Moya y Megías, 2000) constatan los mismos resultados aunque la tendencia es que tanto la escala de de Swim et al. (1995) como la de Tougas et al. (1995) se relacionan con mayor fuerza con la escala de Sexismo Hostil que con la escala de Sexismo Benevolente.

No obstante lo que se evidencia en estas investigaciones es que las escalas de sexismo moderno, como la de Swim et al. (1995) y la de Tougas et al. (1995), miden un sexismo centrado en las relaciones laborales y políticas (medidas de acción positivas) mientras que la escala de Sexismo Ambivalente se sitúa en una dimensión relacional y es la única que conjuga el componente positivo del Sexismo Benevolente con el tradicional. Y son Glick y Fiske (1996) los únicos que fundamentan teóricamente este Sexismo Benevolente que unido al Hostil conforman su teoría del Sexismo Ambivalente.

\section{Actitudes ambivalentes hacia hombres}

Siguiendo la misma lógica argumental, si las complejas relaciones de dependencia/independencia que caracterizan a los sexos determinan la presencia de un sexismo ambivalente hacia las mujeres, también este sexismo ambivalente podría reflejarse hacia los hombres (Glick y Fiske, 1999).

Tradicionalmente los hombres siempre han poseído el poder de las instituciones sociales, políticas y económicas, mientras que las mujeres aunque tenían el poder diádico, siempre han estado subordinadas a los hombres. Esto genera en las mujeres actitudes ambivalentes hacia los hombres, ya que por un lado están resentidas hacia el poder que tienen los hombres, pero por otro, su dependencia puede también proporcionarles incentivos para adular a los hombres. Glick y Fiske (1999) afirman que las mujeres, al igual que los hombres, responden simultáneamente ante el poder estructural de los hombres y el poder diádico de las mujeres, manteniendo creencias hostiles y benevolentes hacia los hombres.

Con respecto a las creencias hostiles los miembros del grupo subordinado, en este caso las mujeres, se resienten ante el poder y el alto estatus que tiene el grupo dominante. Las actitudes hostiles hacia hombres reflejan la jerarquía establecida entre los sexos, se basan en la idea de que los hombres son poseedores de unos rasgos que los ubican en los puestos de poder y dominancia y que además las diferencias entre los géneros son naturales e inevitables (Glick et al., 2004). Aunque hombres y mujeres pertenecen a dos grupos, frecuentemente interactúan, y esto les lleva a las mujeres a experimentar diariamente la fricción que genera la hostilidad hacia hombres. En la investigación de Swim, Hyers, Cohen y Fergunson (2001) con dos muestras, una de alumnas que asumían actitudes más tradicionales hacia los géneros y la otra formada por alumnas con visiones más igualitarias que apoyaban los movimientos de las mujeres, el objetivo fue identificar los incidentes sexistas que habían tenido en una semana. Se encontraron con que las alumnas que asumían visiones más tradiciones hacia las mujeres habían sufrido más incidentes sexistas que las mujeres menos tradicionales. En este sentido Glick et al. (2004) sugiere que las mujeres que asumen roles tradicionales suelen 
experimentar frecuentemente mayores frustraciones en sus interacciones con los hombres, ya que ellos ejercen abiertamente su dominancia sobre ellas, lo que provoca en las mujeres un mayor resentimiento y, en consecuencia, un mayor apoyo a la hostilidad hacia los hombres.

Pero Glick y Fiske (1999) afirman que no es probable que las mujeres sean puramente hostiles en sus actitudes hacia los hombres. Ya que la mayoría de los hombres son dependientes de las mujeres y a su vez la mayoría de las mujeres son también dependientes de los hombres, esto hace que algunas mujeres apoyen y justifiquen la dominación masculina y les lleve a tener actitudes benevolentes hacia los hombres. Otra razón es, que debido a que los hombres suelen recompensar a aquellas mujeres que adoptan roles tradicionales (sexismo benevolente) y suelen castigar a aquellas mujeres que no los cumplen (sexismo hostil), no es de extrañar que muchas mujeres asuman actitudes benevolentes hacia los hombres. Estas mujeres son posiblemente las que desempeñan roles tradicionales de esposa y madre. Además, estas mujeres dependen de los hombres que son los que las mantienen económicamente, y esta dependencia les proporciona fuertes razones para, por un lado, sentir resentimiento y, por otro, respetar el poder de los hombres.

Por consiguiente las mujeres que tienen visiones más tradicionales de los hombres son probablemente las que mantienen actitudes ambivalentes hacia éstos. Estas mujeres atribuyen a los hombres ambos estereotipos: los subjetivamente positivos (actitudes benevolentes) que perciben a los hombres como competitivos, ambiciosos y fuertes; y los estereotipos negativos (actitudes hostiles) que los definen como dominadores, arrogantes y hostiles.

En cuanto a las mujeres no tradicionales como las feministas o las profesionales son las que manifiestan más claramente actitudes hostiles hacia hombres, debido a que perciben el poder de los hombres y la amenaza que quieren ejercer sobre ellas (Glick y Fiske, 1999). Esto está estrechamente relacionado con la idea de que la escala de actitudes hostiles hacia hombres no debería mostrar fuertes convergencias con el Sexismo Hostil y el benevolente, debido a que la hostilidad hacia hombres no justifica las relaciones de género tradicionales. En contraste, la escala de Sexismo Hostil se relaciona con la escala de Benevolencia hacia hombres porque los más sexistas hostiles, que ven a las mujeres como inferiores, deben, al menos por definición, tener actitudes benevolentes hacia los hombres. También las actitudes benevolentes hacia los hombres están relacionadas con el Sexismo Benevolente hacia las mujeres, esto sugiere que las personas sexistas hacia mujeres es probable que tengan actitudes benevolentes hacia los hombres. Por consiguiente, la escala de benevolencia hacia hombres correlaciona con las dos escalas de ASI, pero la escala de hostilidad es esperable que no correlacione con ellas (Glick y Fiske, 1999). Sin embargo en un estudio transcultural posterior Glick et al. (2004) señalan que en caso de que se produzca la relación entre las escalas de sexismo y la hostilidad hacia hombres reflejaría la jerarquía que existe entre los géneros. De manera que en los países donde los hombres asumen actitudes más sexistas hostiles, las mujeres demuestran actitudes más hostiles hacia hombres. Esto coincide con lo que Glick y Fiske (1999) señalaban que la Hostilidad hacia hombres reflejaba el resentimiento de las mujeres hacia la hostilidad de los hombres.

Ambas escalas, la de hostilidad y benevolencia hacia hombres, correlacionan fuertemente, porque en ambas actitudes se presume que los hombres y las mujeres son la mitad que complementan el todo (Glick y Fiske, 1999). Esta correlación positiva es la que indica la existencia de las actitudes ambivalentes hacia los hombres.

\section{La escala AMI}

La Escala de Ambivalencia hacia los Hombres (AMI) fue creada por Glick y Fiske (1999) para medir los prejuicios hostiles y benevolentes que las mujeres tienen hacia los hombres. Glick y Fiske (1999) la definieron como la escala hermana del ASI porque ambas comparten la misma base teórica. Esta escala está formada por 20 ítems que se agrupan en dos factores: el primero fue denominado Hostilidad hacia los hombres y el segundo Benevolencia hacia los hombres. Cada uno de estas dimensiones se divide a su vez en tres subfactores que están relacionados con el poder estructural masculino, la diferenciación de género y la sexualidad al igual que en la escala de Sexismo Ambivalente (ASI). La Hostilidad hacia hombres se divide en tres subfactores: el Resentimiento Parter- 
nalista que implica que los miembros del grupo subordinado, en este caso las mujeres, pueden tener resentimiento hacia el poder y el alto estatus del grupo dominante, los hombres; la Diferenciación de Género Compensatoria que permite a las mujeres diferenciarse positivamente de los hombres; y la Hostilidad Heterosexual que supone asumir que el hombre ejerce un papel dominante en las distintas situaciones, hasta el punto de poder incluso agredir sexualmente a una mujer. El conocimiento por parte de las mujeres de esta amenaza ha mostrado tener efectos importantes como tener un resentimiento considerable hacia los hombres (Glick y Fiske, 1999). La escala de Benevolencia hacia hombres se subdivide en otros tres factores: el Maternalismo que parte de la dependencia entre sexos para la reproducción y las relaciones románticas; la Diferenciación de Género Complementaria que implica que el grupo de bajo poder, en este caso el de las mujeres, debe creer que ellas son menos ambiciosas, dominantes, inteligentes que el grupo dominante, que son los hombres. Esta benevolencia hacia los hombres proviene de la admiración que las mujeres tienen hacia el alto estatus de los hombres; y la Intimidad Heterosexual que es entendida como la "necesidad" que una mujer tiene de un hombre.

Posteriormente Glick y su equipo de investigación validaron la escala AMI en un estudio transcultural en el que contaron con 8.360 participantes de 16 naciones (Glick et al., 2004). En la mayoría de los países se confirma el modelo factorial completo (Hostilidad con sus tres subfactores y Benevolencia con sus tres subfactores), exceptuando a Perú. No obstante, diversos estudios nacionales solo confirman la existencia de dos factores de Hostilidad y de Benevolencia hacia hombres (Lameiras et al. 2001; Lameiras y Rodríguez, 2002; 2003; Lameiras et al., 2004 y Rodríguez, 2006). Las fiabilidades obtenidas en las subescalas de Hostilidad y Benevolencia hacia hombres son altas, están entre .75 y .90 (Glick y Fiske, 1999, Glick et al., 2004).

Analizando las diferencias de género dentro de cada nación, se encontró que las mujeres en la mayoría de los países, excepto en Inglaterra, eran más hostiles hacia los hombres; mientras que los hombres por su parte tenían actitudes más benevolentes hacia los hombres. Aunque no se detectaron diferencias significativas entre hombres y mujeres en sus niveles de Benevolencia hacia hombres en países como Holanda, México, Portugal, España y Taiwán (Glick et al., 2004).

En cuanto a las relaciones entre la escala ASI y AMI en este estudio transcultural señalar que las diferencias de género en las puntuaciones de hostilidad hacia hombres fueron relacionadas de forma significativa y negativa. Esto quiere decir que las naciones donde los hombres apoyan más el Sexismo Hostil las mujeres, por su parte, muestran actitudes más hostiles hacia hombres. Tal y como predecía Glick y Fiske (1999), la hostilidad hacia los hombres refleja una parte del resentimiento de las mujeres hacia la hostilidad que los hombres demuestran hacia las mujeres. De esta forma, la hostilidad hacia hombres de las mujeres se incrementa de tal modo que su tendencia a apoyar las actitudes hostiles se da con mayor fuerza que en los hombres.

Solamente una correlación significativa implica las diferencias de género en la escala de Sexismo Benevolente. Este resultado ya se había confirmado en el estudio transcultural anterior (Glick et al., 2000) que el mayor apoyo de los hombres al Sexismo Hostil o al Sexismo Benevolente provoca el mayor apoyo de las mujeres a las actitudes Benevolentes hacia hombres. Cuando la mayoría de la gente de una nación tienen actitudes más tradicionales hacia los roles de los géneros, la mayoría de las mujeres optaban por apoyar las actitudes Benevolentes hacia los hombres, aunque también actitudes más Hostiles hacia hombres. De esta forma las mujeres de naciones más tradicionales pueden tener un mayor resentimiento hacia los hombres, porque posiblemente son vistos como abusadores del poder que tienen, pero este resentimiento no implica necesariamente un cambio de la jerarquía establecida socialmente entre los géneros y coexiste con las creencias benevolentes hacia el rol de los hombres como proveedores del sustento económico y como protectores de las mujeres.

Está claro que en los hombres apoyar las actitudes benevolentes hacia ellos tiene beneficios, mientras que en las mujeres apoyar el Sexismo Benevolente les ocasiona tanto costes como beneficios. Los costes implican que la mujer siempre estaría subordinada al hombres y los beneficios conllevan la protección, la provisión y el afecto a causa de las pocas oportunidades que tienen las mujeres para conseguir la independencia económica y social (Glick et al., 2004). 


\section{Escala AMI y otras medidas de sexismo}

Glick y Fiske (1999) afirman que la escala AMI es el único instrumento de medida útil para evaluar las actitudes ambivalentes que las mujeres tienen hacia los hombres. Ya que las demás escalas de actitudes hacia hombres como la escala de Actitudes hacia los hombres de Downs y Engleson (1982), y la escala de Actitudes hacia los hombres de Iazzo (1983) son escalas que miden visiones tradicionales de los hombres en distintos ámbitos como el trabajo, la familia y la sexualidad.

Glick y Fiske (1999) apuntan que existe una fuerte correlación entre las escalas de Iazzo (1983) y de Downs y Engleson (1982) y su escala AMI (y también con las subescalas de hostilidad y benevolencia hacia hombres), pero correlacionan en el mismo sentido. Es decir, si la escala de Iazzo mide actitudes en sentido positivo y negativo hacia hombres, entonces las subescalas de hostilidad y de benevolencia deberían mostrar correlaciones opuestas con esta escala; con la subescala de hostilidad en sentido positivo y con la subescala de benevolencia en sentido negativo. Pero esto no es así, lo que le sugiere a los autores que a pesar de que estas escalas tienen determinada similitud con la escala AMI (Glick y Fiske, 1999), en ellas no se distingue simultáneamente entre actitudes tradicionales de los hombres que son subjetivamente positivas (actitudes benevolentes) y aquellas que son negativas (actitudes hostiles).

Es importante matizar los giros teóricos sobre la relación de las escalas de Sexismo Ambivalente (ASI) y de Ambivalencia hacia hombres (AMI). Hay que recordar que en el estudio en el que se elaboró la escala AMI, Glick y Fiske (1999) apoyaban que no existía relación entre las escalas del Sexismo Ambivalente (ASI) con la escala de Hostilidad hacia hombres del AMI, y era explicado debido a que la hostilidad hacia hombres no justificaba la actitudes tradicionales hacia los géneros. Los resultados del estudio transcultural (Glick et al., 2004), a la vista de que en todos los países se establecía la relación de las escalas del ASI con la Hostilidad hacia hombres (incluso llevando a cabo las correlaciones parciales), llevan a Glick y su equipo de investigación a justificarlo argumentando que esa relación expresa la jerarquía que se da entre los géneros. Diversos estudios constatan la existencia de la relación entre ambas escalas, y en concreto en la relación entre la Hostilidad hacia hombres y las escalas del ASI (Lameiras et al. 2001; Lameiras y Rodríguez, 2002; 2003; y Lameiras et al., 2004).

En suma, la teoría del Sexismo Ambivalente tanto hacia mujeres como hacia hombres supone un avance importante para conseguir la "igualdad real” entre hombres y mujeres, ya que por medio de sus escalas se pueden identificar las actitudes sexistas benevolentes tan perjudiciales para las mujeres, para poder combatirlas. Y así conseguir una realidad más igualitaria y simétrica entre los géneros.

\section{Referencias}

Allport, G. W. (1954). The Nature of Prejudice. Reading, MA: Addison-Wesley.

Bulh, C., Hanges, P., Sipe, W., \& Salvaggio, A. (1999). The search for ambivalent sexism: A comparision of two measures. Educational and Psychological Measurement, 59 (6), 898-909.

Downs, A. C., \& Engleson, S. A. (1982). The Atittudes toward men Scale (AMS):An analysis of the role and status of men and masculinity. Catalog of Selectd Documents in Psychology, 12 (4), 45.

Eagly, A. H., \& Mladinic, A. (1993). Are people prejudiced against women?. Some answers from research on attitudes, gender stereotypes and judgments of competence. En D.M. W. Stroebe \& M. Hewstone (Eds.), European Review of Social Psychology (vol. 5, pp. 1-35). New York: Wiley.

Expósito, F., \& Moya, M., \& Glick, P. (1998). Sexismo ambivalente: medición y correlatos. Revista de Psicología Social, 55, 893-905. 
Frese, B., Moya, M., \& Megías, J. (2000). Actitudes hacia las víctimas de agresiones sexuales y probabilidad de actuar como los agresores. En A. Ovejero, M. Moral \& P. Vivas (Eds.), Aplicaciones en psicología social (pp. 112-117). Madrid: Biblioteca Nueva.

Glick, P., \& Fiske, S. T. (1996). The Ambivalent Sexism lnventory: Differentiating hostile and benevolen sexism. Journal of Personality and Social Psychology, 70, 491-512.

Glick, P., \& Fiske, S. T. (1999). The Ambivalence toward Men Inventory: Differentiating hostile and benevolent beliefs about men. Psychology of Women Quarterly, 23, 519-536.

Glick, P., Diebold, J., Bailey-Werner, B., \& Zhu, L. (1997). The two faces of Adam:Ambivalent sexism and polarized attitudes toward women. Personality and Social psychology Bulletin, 23, 1323-1334.

Glick, P., Fiske, S. T., Mladinic, A., Saiz, J. L., Abrams D., Masser, B., Adetoun, B.,Osagie, J. E., Akande, A., Alao, A., Brunner, A., Willemensen, T. M., Chipeta, K., Dardanne, B., Dijksterhuis, A., Wigbouldus, D., Eckes, T., Six-Materna, Y., Expósito, F., Moya, M., Foddy, M., Kim, H. J. Lameiras, M., Sotelo, M. J., Mucchi-Faina, A.,Romani, M., Sakalli, N., Udegbe, B., Yamamoto, M., Ui, M., Ferreira, M. C. (2000). Beyond Prejudice as Simple Antipathy: Hostile and Benevolent Sexism Across Cultures. Journal of Personality and Social Psyohology, 79, 763-775.

Glick, P., Lameiras, M., Fiske, S. T., Masser, B., Volpato, C., Manganelli, A. M., Pek, J.C.X., Huang, L., Sakalli-Urgurlu, N., Rodríguez, Y., D’Avila, M.L., Willemsen, T. M., Brunner, A., Six-Materna, I., \& Wells, R. (2004). Bad but Bold: Ambivalent Attitudes toward Men Predict Gender Inequality in 16 Nations. Journal of Personality and Social Psychology, 86, 5, 713-728.

Iazzo, A.N. (1983). The construction and validation of Atittudes Toward Men Scale. Psychological Record, 33. 371-378.

Katz, I. (1981). Stigma: A social psychological analysis. Hillsdale, L.: Erlbaum.

Katz, I., \& Hass, R. C. (1988). Racial ambivalence and American value conflict: Correlational and priming studies of dual cognitive structure. Journal of Personality and Social Psychology, 55, 893-905.

Lameiras M., Rodríguez, Y., \& Sotelo, M. J. (2001). Sexism and Racism in a Spanish Sample of Secondary School Students. Social Indicators Research, 54 (3), 309-328.

Lameiras, M., \& Rodríguez, Y. (2002). Evaluación del sexismo moderno en adolescentes. Revista de Psicología Social, 17 (2), 119-127.

Lameiras, M., \& Rodríguez, Y. (2003). Evaluación del sexismo ambivalente en estudiantes gallegos/ as. Revista de Acción Psicológica, 2 (2), 131-136.

Lameiras, M., Rodríguez, Y., \& González, M. (2004). Evolution of Hostil Sexism and Benevolent Sexism in a Spanish Sample. Social Indicators Research, 66, 197-211.

Lameiras, M., Rodríguez, Y., Carrera, M. V., \& Calado Otero, M. (2006). Profesoras e profesores no sistema universitario galego. Unha perspectiva de Xénero. Santiago: Servicio de Igualdade, Xunta de Galicia.

Masser, B., \& Abrams, D. (1999). Contemporary sexism. The relationships among Hostility, Benevolence and Neosexism. Psychology of Women Quarterly, 6, 503-517.

McHugh, C. M., \& Frieze, H. I. (1997). The measurement of gender-role attitudes. A review and commentary. Psychology of Women Quarterly, 21, 1-16.

Moya, M., Navas, M., \& Gómez Berrocal, C. (1991). Escala sobre ideología del rol sexual. Libro de comunicaciones del III Congreso Nacional de Psicología Social, 1, (pp.554-566). Santiago de Compostela.

Moya, M., Páez, D., Glick, P., Fernández, I., \& Poeschl, G. (2001). Sexismo, masculinidad-feminidad 
y factores culturales. Revista Electrónica de Motivación y Emoción, 8/9.

Rodríguez, Y.C. (2006). Evaluación de las actitudes sexistas en la comunidad educativa española. Universidad de Vigo: Tesis doctoral (no publicada).

Sears, D.O. (1988). Symbolic racism. En P. A. Katz \& D. A. Taylor (Eds.), Eliminating racism (pp. 53-84). New York: Plenum Press.

Spence, J. T., \& Helmreich, R. L. (1972). The Attitudes Toward Women Scale: An objective instrument to measure the attitude toward the rights and roles women in contemporary society. JSAS: Catalog of Selected Documents in Psychology, 2, 66-67.

Swim, J. K., Aikin, W.S., HaII, W. S., \& Hunter, B. A. (1995). Sexisn, and Racism: Old-fashioned and Modern Prejudices. Journal of Personality and Social Psychology, 68, 2, 199-214.

Swim, J. K., Hyers, L. L., Cohen, L. L., \& Fergunson, M. J. (2001). Every day sexism: Evidence for its incidence, nature, and psychological impact form three diary studies. Journal of Social Issues, 57, 31-53.

Tougas, F., Brown, A. M, Beaton, \& Joly, S. (1995). Neosexism: Plus Ca Change, Plus C`est Pareil. Journal of Personality and Social Psychology, 21, 842-849. 